\title{
Influence Function of Multiple Kernel Canonical Analysis to Identify Outliers in Imaging Genetics Data
}

\author{
Md. Ashad Alam \\ Department of Biomedical \\ Engineering \\ Tulane University \\ New Orleans, LA 70118, USA \\ malam@tulane.edu
}

\author{
Vince Calhoun ${ }^{\dagger}$ \\ Department of Electrical and \\ Computer Engineering \\ The University of New Mexico \\ Albuquerque, NM 87131, USA \\ vcalhoun@mrn.org
}

\author{
Yu-Ping Wang \\ Department of Biomedical \\ Engineering \\ Tulane University \\ New Orleans, LA 70118,USA \\ wyp@tulane.edu
}

\begin{abstract}
Imaging genetic research has essentially focused on discovering unique and co-association effects, but typically ignoring to identify outliers or atypical objects in genetic as well as non-genetics variables. Identifying significant outliers is an essential and challenging issue for imaging genetics and multiple sources data analysis. Therefore, we need to examine for transcription errors of identified outliers. First, we address the influence function (IF) of kernel mean element, kernel covariance operator, kernel cross-covariance operator, kernel canonical correlation analysis (kernel CCA) and multiple kernel CCA. Second, we propose an IF of multiple kernel CCA, which can be applied for more than two datasets. Third, we propose a visualization method to detect influential observations of multiple sources of data based on the IF of kernel CCA and multiple kernel CCA. Finally, the proposed methods are capable of analyzing outliers of subjects usually found in biomedical applications, in which the number of dimension is large. To examine the outliers, we use the stem-and-leaf display. Experiments on both synthesized and imaging genetics data (e.g., SNP, fMRI, and DNA methylation) demonstrate that the proposed visualization can be applied effectively.
\end{abstract}

\section{Categories and Subject Descriptors}

[Machine learning theory]: Kernel methods; [Robustness]: Redundancy; [Information integration]: Mediators and data integration

\section{General Terms}

Theory and Performance

\footnotetext{
*Department of Statistics, Hajee Mohammad Danesh Science and Technology University, Dinajpur 5200, Bangladesh. ${ }^{\dagger}$ Image Analysis and MR Research, The Mind Research Network.

Permission to make digital or hard copies of all or part of this work for personal or classroom use is granted without fee provided that copies are not made or distributed for profit or commercial advantage and that copies bear this notice and the full citation on the first page. Copyrights for components of this work owned by others than ACM must be honored. Abstracting with credit is permitted. To copy otherwise, or republish, to post on servers or to redistribute to lists, requires prior specific permission and/or a fee. Request permissions from Permissions@ acm.org.

BCB'16, October 2-5, 2016, Seattle, WA, USA.

(C) 2016 ACM. ISBN 978-1-4503-4225-4/16/10 ...\$15.00.

DOI: http://dx.doi.org//10.1145/2975167.2975189.
}

\section{Keywords}

Kernel CCA, Multiple kernel CCA, Influence function, Outlier detection in imaging genetics, Data integration

\section{INTRODUCTION}

Identifying significant outliers is an essential and challenging issue in statistical machine learning for multiple sources data analysis. The atypical objects or outliers, data that cause surprise in relation to the majority of the data, often occur in the real data. Outliers may be right, but we need to examine for transcription errors. They can play havoc with classical statistical methods [20]. Once a statistical approach is applied to imaging genetics data containing outliers, the results can be deceptive with high probability. To overcome this problem, since 1960 many robust methods have been developed, which are less sensitive to outliers. The goals of robust statistics are to use the methods from the bulk of the data and identify the points deviating from the original patterns for further investment [29, 24, 33]. But it is well-known that most robust methods are computationally intensive and have the curse of dimensionality problem. The outliers need to be removed or downweighted prior to fitting non-robust statistical or machine learning approaches $[16$, $34,37]$.

The incorporation of various unsupervised learning methods into genomic analysis is a rather recent topic. Using the dual representations in problems of supervised and unsupervised learning, the task of learning from multiple data sources is related to the kernel-based data integration, which has been actively studied in the last decade [26, 2]. Kernel fusion in unsupervised learning has a close connection with unsupervised kernel methods. As unsupervised kernel methods, kernel principal component analysis [39, 4], kernel canonical correlation analysis $[1,5,3]$, weighted multiple kernel CCA have been extensively studied for decades [38]. But these methods are not robust; they are sensitive to contaminated data. To apply all of these non-robust methods, for instance in genomics, outliers identification and/ or robust approaches are essential.

Due to the properties of eigen decomposition, kernel CCA is still a well-applied method for multiple sources data analysis and integration. An empirical comparison and sensitivity analysis for robust linear CCA and kernel CCA were also discussed, which give similar interpretation as kernel PCA without any theoretical results [8, 7]. In addition, [36] and [6] have proposed the IF of canonical correlation and ker- 
nel CCA but the IF of multiple kernel CCA has not been studied. All of these considerations motivate us to conduct studies on the IF of multiple kernel CCA to identify outliers in imaging genetics data sets: SNP, fMRI, and DNA methylation.

The contribution of this paper is fourfold. We address the IF of kernel mean element (kernel ME), kernel covariance operator (kernel CO), kernel cross-covariance operator (kernel CCO), kernel canonical correlation analysis (kernel CCA) and multiple kernel CCA. After that, we propose the IF of multiple kernel CCA, which can be applied for more than two datasets. Based on this results, we propose a visualization method to detect influential observations of multiple sources data based. The proposed method is capable of analyzing the outliers usually found in biomedical application, in which the number of dimension is large. To confirm the outliers, we use the step-and-leaf display. The results imply that the proposed method enables to identify outliers in synthesized and imaging genetics data (e.g., SNP, fMRI, and DNA methylation).

The remainder of the paper is organized as follows. In the next section, we provide a brief review of kernel ME, kernel $\mathrm{CO}$, and kernel CCO. In Section 3, we discuss in brief the IF, IF of kernel ME and IF of kernel CO. After a brief review of kernel CCA in Section 2.4, we propose the IF of classical multiple kernel CCA in Section 3. In Section 5, we describe experiments conducted on both synthesized and real data analysis from an imaging genetics study with a visualizing method.

\section{PRELIMINARY}

Kernel ME, kernel $\mathrm{CO}$ and kernel CCO with positive definite kernel have been extensively applied to nonparametric statistical inference through representing distribution in the form of means and covariance in RKHS [22, 18, 40, 30, 21]. Basic notations of kernel MEs, kernel CO and kernel CCO with their robustness through IF are briefly discussed below.

\subsection{Kernel mean element}

Let $F_{X}, F_{Y}$ and $F_{X Y}$ be the probability measure on $\mathcal{X}, \mathcal{Y}$ and $\mathcal{X} \times \mathcal{Y}$, respectively. Also let $X_{1}, X_{2}, \ldots, X_{n}, ; Y_{1}, Y_{2}, \ldots, Y_{n}$ and $\left(X_{1}, Y_{1}\right),\left(X_{2}, Y_{2}\right), \ldots,\left(X_{2}, Y_{2}\right)$ be the random sample from the respective distribution. A symmetric kernel $k(\cdot, \cdot)$ defined on a space is called positive definite kernel if the Gram matrix $\left(k\left(X_{i}, X_{j}\right)\right)_{i j}$ is positive semi-definite [10]. By the reproduction properties and kernel trick, the kernel can evaluate the inner product of any two feature vectors efficiently without knowing an explicit form of either the feature map $(\Phi(\cdot)=k(\cdot, X), \forall X \in \mathcal{X})$ or feature space $(\mathcal{H})$. In addition, the computational cost does not depend on the dimension of the original space after computing the Gram matrices $[19,4]$. A mapping $\mathcal{M}_{X}:=\mathbb{E}_{X}[\Phi(X)]=\mathbb{E}_{X}[k(\cdot, X)]$ with $\mathbb{E}_{X}[\sqrt{k(X, X)}]<\infty$ is an element of the RKHS $\mathcal{H}_{X}$. By the reproducing property with $X \in \mathcal{X}$, kernel mean element is defined as

$$
\left\langle\mathcal{M}_{X}, f\right\rangle_{\mathcal{H}_{X}}=\left\langle\mathbb{E}_{X}[k(\cdot, X)], f\right\rangle_{\mathcal{H}_{X}}=\mathbb{E}_{X}[f(X)],
$$

for all $f \in \mathcal{H}_{X}$. Given an independent and identically distributed sample, the mapping $m_{X}=\frac{1}{n} \sum_{i=1}^{n} \Phi\left(X_{i}\right)=$ $\frac{1}{n} \sum_{i=1}^{n} k\left(\cdot, X_{i}\right)$ is an empirical element of the RKHS, $\mathcal{H}_{X}$, $\left\langle m_{X}, f\right\rangle_{\mathcal{H}_{X}}=\left\langle\frac{1}{n} \sum_{i=1}^{n} k\left(\cdot, X_{i}\right), f\right\rangle=\frac{1}{n} \sum_{i=1}^{n} f\left(X_{i}\right)$.

\subsection{Kernel covariance operator}

By the reproducing property, kernel CCO, $\Sigma_{X Y}:=\mathcal{H}_{Y} \rightarrow$ $\mathcal{H}_{X}$ with $\mathbb{E}_{X}\left[k_{X}(X, X)\right]<\infty$, and $\mathbb{E}_{Y}\left[k_{Y}(Y, Y)\right]<\infty$ is defined as

$$
\begin{aligned}
& \left\langle f_{X}, \Sigma_{X Y} f_{Y}\right\rangle_{\mathcal{H}_{X}}= \\
& \mathbb{E}_{X Y}\left[\left\langle f_{X}, k_{X}(\cdot, X)-\mathcal{M}_{X}\right\rangle_{\mathcal{H}_{X}}\left\langle f_{Y}, k_{Y}(\cdot, Y)-\mathcal{M}_{Y}\right\rangle_{\mathcal{H}_{Y}}\right]= \\
& \mathbb{E}_{X Y}\left[\left(f_{X}(X)-E_{X}[f(X)]\right)\left(f_{Y}(Y)-E_{Y}[f(Y)]\right)\right] .
\end{aligned}
$$

\subsection{Influence function of kernel operators}

To define the notation of robustness in statistics, different approaches have been proposed, for examples, the minimax approach [28], the sensitivity curve [42], the influence functions [23, 25] and in the finite sample breakdown point [15]. Due to its simplicity, the IF is the most useful approach in statistical supervised learning $[14,13]$. In this section, we briefly discuss the notations of IF, IF of kernel ME, and IF of kernel $\mathrm{CO}$ and kernel CCO.

Let $(\Omega, \mathcal{A})$ be a probability space and $(\mathcal{X}, \mathcal{B})$ a measure space. We want to estimate the parameter $\theta \in \Theta$ of a distribution $F$ in $\mathcal{A}$. We assume that there exists a functional $R: \mathcal{D}(R) \rightarrow \mathbb{R}$, where $\mathcal{D}(R)$ is the set of all probability distribution in $\mathcal{A}$. Let $G$ be a distribution in $\mathcal{A}$. If data do not fallow the model $F$ exactly but slightly going toward $G$, the Gâteaux Derivative at $F$ is called influence function [30]. The IF of complicated statistics, which is a function of simple statistics, can be calculated with the chain rule, Say $R(F)=a\left(R_{1}(F), \ldots ., R_{s}(F)\right)$. Specifically,

$$
I F_{R}(z)=\sum_{i=1}^{s} \frac{\partial a}{\partial R_{i}} I F_{R_{i}}(z) .
$$

It can also be used to find the IF of a transformed statistic, given the influence function for the statistic itself.

The IF of kernel CCO, $R\left(F_{X Y}\right)$, with joint distribution, $F_{X Y}$, using complicated statistics at $Z^{\prime}=\left(X^{\prime}, Y^{\prime}\right)$ is given by

$$
\begin{aligned}
& \operatorname{IF}\left(\cdot, \mathrm{Z}^{\prime}, \mathrm{R}, \mathrm{F}_{\mathrm{XY}}\right) \\
& =\left\langle k_{X}\left(\cdot, X^{\prime}\right)-\mathcal{M}\left[F_{X}\right], f\right\rangle_{\mathcal{H}_{X}}\left\langle k_{Y}\left(\cdot, Y^{\prime}\right) \mathcal{M}\left[F_{Y}\right], g\right\rangle_{\mathcal{H}_{Y}} \\
& -\mathbb{E}_{X Y}\left[\left\langle k_{X}(\cdot, X)-\mathcal{M}\left[F_{X}\right], f\right\rangle_{\mathcal{H}_{X}}\left\langle k_{Y}(\cdot, Y)-\mathcal{M}\left[F_{Y}\right], g\right\rangle_{\mathcal{H}_{Y}}\right]
\end{aligned}
$$

which is estimated with the data points $\left(X_{1} Y_{1}\right),\left(X_{2}, Y_{2}\right), \cdots$, $\left(X_{n}, Y_{n}\right) \in \mathcal{X} \times \mathcal{Y}$ for every $Z_{i}=\left(X_{i}, Y_{i}\right)$ as

$$
\begin{gathered}
\widehat{\operatorname{IF}}\left(Z_{i}, Z^{\prime}, R, F_{X Y}\right) \\
=\left[k_{X}\left(X_{i}, X^{\prime}\right)-\frac{1}{n} \sum_{b=1}^{n} k_{X}\left(X_{i}, X_{b}\right)\right]\left[k_{Y}\left(Y_{i}, Y^{\prime}\right)\right. \\
\left.-\frac{1}{n} \sum_{b=1}^{n} k_{Y}\left(Y_{i}, Y_{b}\right)\right]-\frac{1}{n} \sum_{d=1}^{n}\left[k_{X}\left(X_{i}, X_{d}\right)-\frac{1}{n} \sum_{b=1}^{n} k_{X}\left(X_{i}, X_{b}\right)\right] \\
{\left[k_{Y}\left(Y_{i}, Y_{d}\right)-\frac{1}{n} \sum_{b=1}^{n} k_{Y}\left(Y_{i}, Y_{b}\right)\right] .}
\end{gathered}
$$

For the bounded kernels, the above IFs have three properties: gross error sensitivity, local shift sensitivity and rejection point. These are not true for the unbounded kernels, for example, liner and polynomial kernels. We are able to make similar conclusion for the kernel $\mathrm{CO}$ and kernel CCO. Most of the unsupervised methods explicitly or implicitly depend on the kernel CO or kernel CCO. They are sensitive 
to contaminated data, even when using the bounded positive definite kernels. To overcome the problem, the outliers need to removed from the data.

\subsection{Kernel CCA}

In this section, we review the kernel CCA, the IF and empirical IF (EIF) of kernel CCA. After that we address the multiple kernel CCA and proposed the IF and EIF of multiple kernel CCA based on the IF of kernel CO and kernel CCO.

The aim of kernel $C C A$ is to seek two sets of functions in the RKHS for which the correlation (Corr) of random variables is maximized. Given two sets of random variables $X$ and $Y$ with two functions in the RKHS, $f_{X}(\cdot) \in \mathcal{H}_{X}$ and $f_{Y}(\cdot) \in \mathcal{H}_{Y}$, the optimization problem of the random variables $f_{X}(X)$ and $f_{Y}(Y)$ is

$$
\max _{\substack{f_{X} \in \mathcal{H}_{X}, f_{Y} \in \mathcal{H}_{Y} \\ f_{X} \neq 0, f_{Y} \neq 0}} \operatorname{Corr}\left(f_{X}(X), f_{Y}(Y)\right)
$$

The optimizing functions $f_{X}(\cdot)$ and $f_{Y}(\cdot)$ are determined up to scale.

Using a finite sample, we are able to estimate the desired functions. Given an i.i.d sample, $\left(X_{i}, Y_{i}\right)_{i=1}^{n}$ from a joint distribution $F_{X Y}$, by taking the inner products with elements or "parameters" in the RKHS, we have features $f_{X}(\cdot)=\left\langle f_{X}, \Phi_{X}(X)\right\rangle_{\mathcal{H}_{X}}=\sum_{i=1}^{n} a_{X}^{i} k_{X}\left(\cdot, X_{i}\right)$ and $f_{Y}(\cdot)=$ $\left\langle f_{Y}, \phi_{Y}(Y)\right\rangle_{\mathcal{H}_{Y}}=\sum_{i=1}^{n} a_{Y}^{i} k_{Y}\left(\cdot, Y_{i}\right)$, where $k_{X}(\cdot, X)$ and $k_{Y}(\cdot, Y)$ are the associated kernel functions for $\mathcal{H}_{X}$ and $\mathcal{H}_{Y}$, respectively. The kernel Gram matrices are defined as $\mathbf{K}_{X}:=$ $\left(k_{X}\left(X_{i}, X_{j}\right)\right)_{i, j=1}^{n}$ and $\mathbf{K}_{Y}:=\left(k_{Y}\left(Y_{i}, Y_{j}\right)\right)_{i, j=1}^{n}$. We need the centered kernel Gram matrices $\mathbf{M}_{X}=\mathbf{C} \mathbf{K}_{X} \mathbf{C}$ and $\mathbf{M}_{Y}=$ $\mathbf{C K}_{Y} \mathbf{C}$, where $\mathbf{C}=\mathbf{I}_{n}-\frac{1}{n} \mathbf{B}_{n}$ with $\mathbf{B}_{n}=\mathbf{1}_{n} \mathbf{1}_{n}^{T}$ and $\mathbf{1}_{n}$ is the vector with $n$ ones. The empirical estimate of Eq. (1) is then given by

$$
\max _{\substack{f_{X} \in \mathcal{H}_{X}, f_{Y} \in \mathcal{H}_{Y} \\ f_{X} \neq 0, f_{Y} \neq 0}} \frac{\widehat{\operatorname{Cov}}\left(f_{X}(X), f_{Y}(Y)\right)}{\left[\widehat{\operatorname{Var}}\left(f_{X}(X)\right)\right]^{1 / 2}\left[\widehat{\operatorname{Var}}\left(f_{Y}(Y)\right)\right]^{1 / 2}}
$$

where

$$
\begin{aligned}
& \widehat{\operatorname{Cov}}\left(f_{X}(X), f_{Y}(Y)\right)=\frac{1}{n} \mathbf{a}_{X}^{T} \mathbf{M}_{X} \mathbf{M}_{Y} \mathbf{a}_{Y} \\
& \widehat{\operatorname{Var}}\left(f_{X}(X)\right)=\frac{1}{n} \mathbf{a}_{X}^{T} \mathbf{M}_{X}^{2} \mathbf{a}_{X} \\
& \widehat{\operatorname{Var}}\left(f_{Y}(Y)\right)=\frac{1}{n} \mathbf{a}_{Y}^{T} \mathbf{M}_{Y}^{2} \mathbf{a}_{Y},
\end{aligned}
$$

where $\mathbf{a}_{X}$ and $\mathbf{a}_{Y}$ are the directions of $X$ and $Y$, respectively.

$$
\begin{aligned}
& {\left[\begin{array}{cc}
0 & \mathbf{M}_{1} \mathbf{M}_{2} \\
\mathbf{M}_{2} \mathbf{M}_{1} & 0
\end{array}\right]\left[\begin{array}{l}
\mathbf{a}_{X} \\
\mathbf{a}_{Y}
\end{array}\right]=} \\
& \rho\left[\begin{array}{cc}
\mathbf{M}_{1} \mathbf{M}_{1} & 0 \\
0 & \mathbf{M}_{2} \mathbf{M}_{2}
\end{array}\right]\left[\begin{array}{l}
\mathbf{a}_{X} \\
\mathbf{a}_{Y}
\end{array}\right]
\end{aligned}
$$

Unfortunately, the naive kernelization (2) of CCA is trivial and non-zero solutions of generalized eigenvalue problem are $\rho= \pm 1[8,11]$. To overcome this problem, we introduce small regularization terms in the denominator of the right hand side of (2) as

$$
\begin{array}{r}
{\left[\begin{array}{cc}
0 & \mathbf{M}_{1} \mathbf{M}_{2} \\
\mathbf{M}_{2} \mathbf{M}_{1} & 0
\end{array}\right]\left[\begin{array}{l}
\mathbf{a}_{X} \\
\mathbf{a}_{Y}
\end{array}\right]=} \\
\rho\left[\begin{array}{cc}
\left(\mathbf{M}_{1}+\kappa I\right)^{2} & 0 \\
0 & \left(\mathbf{M}_{2}+\kappa I\right)^{2}
\end{array}\right]\left[\begin{array}{l}
\mathbf{a}_{X} \\
\mathbf{a}_{Y}
\end{array}\right]
\end{array}
$$

where the small regularized coefficient is $\kappa>0$.

Using the IF of kernel mean element and covariance operator in the eigenvalue problem in Eq. (3), as shown in [6], the influence function of kernel canonical correlation (kernel CCA ) and kernel canonical variate at $Z^{\prime}=\left(X^{\prime}, Y^{\prime}\right)$ is given by

$$
\begin{gathered}
\operatorname{IF}\left(\mathrm{Z}^{\prime}, \rho_{\mathrm{j}}^{2}\right)=-\rho_{\mathrm{j}}^{2} \overline{\mathrm{f}}_{\mathrm{jX}}^{2}\left(\mathrm{X}^{\prime}\right)+2 \rho_{\mathrm{j}} \overline{\mathrm{f}}_{\mathrm{jX}}\left(\mathrm{X}^{\prime}\right) \overline{\mathrm{f}}_{\mathrm{j} \mathrm{Y}}\left(\mathrm{Y}^{\prime}\right)-\rho_{\mathrm{j}}^{2} \overline{\mathrm{f}}_{\mathrm{jY}}^{2}\left(\mathrm{Y}^{\prime}\right), \\
\operatorname{IF}\left(\cdot, \mathrm{Z}^{\prime}, \mathrm{f}_{\mathrm{jX}}\right)=-\rho_{\mathrm{j}}\left(\overline{\mathrm{f}}_{\mathrm{j} \mathrm{Y}}\left(\mathrm{Y}^{\prime}\right)-\rho_{\mathrm{j}} \overline{\mathrm{f}}_{\mathrm{jX}}\left(\mathrm{X}^{\prime}\right)\right) \mathbb{L} \tilde{\mathrm{k}}\left(\cdot, \mathrm{X}^{\prime}\right)-\left(\overline{\mathrm{f}}_{\mathrm{jX}}\left(\mathrm{X}^{\prime}\right)\right. \\
\left.-\rho_{j} \bar{f}_{j Y}\left(Y^{\prime}\right)\right) \mathbb{L} \Sigma_{X Y} \Sigma_{Y Y}^{-1} \tilde{k}_{Y}\left(\cdot, Y^{\prime}\right)+\frac{1}{2}\left[1-\bar{f}_{j X}^{2}\left(X^{\prime}\right)\right] f_{j X},
\end{gathered}
$$

where $\mathbb{L}=\Sigma_{X X}^{-\frac{1}{2}}\left(\Sigma_{X X}^{-\frac{1}{2}} \Sigma_{X Y} \Sigma_{Y Y}^{-1} \Sigma_{Y X} \Sigma_{X X}^{-\frac{1}{2}}-\rho^{2} \mathbf{I}\right)^{-1} \Sigma_{X X}^{-\frac{1}{2}}$ and similar for the kernel $\mathrm{CV}$ of $f_{Y}, \operatorname{IF}\left(\cdot, \mathrm{Z}^{\prime}, \mathrm{f}_{\mathrm{j} Y}\right)$ It is known that the inverse of an operator may not exit or even exist but may not be continuous in general [17]. While we can derive kernel canonical correlation using correlation operator $\mathbf{V}_{Y X}=\Sigma_{Y Y}^{-\frac{1}{2}} \Sigma_{Y X} \Sigma_{X X}^{-\frac{1}{2}}$, even when $\Sigma_{X X}^{-\frac{1}{2}}$ and $\Sigma_{Y Y}^{-\frac{1}{2}}$ are not proper operators, the IF of covariance operator is true only for the finite dimensional RKHSs. For infinite dimensional RKHSs, we can find IF of $\Sigma_{X X}^{-\frac{1}{2}}$ by introducing a regularization term as follows

$$
\begin{aligned}
\operatorname{IF}\left(\cdot, \mathrm{X}^{\prime},\left(\Sigma_{\mathrm{XX}}+\kappa \mathbf{I}\right)^{-\frac{1}{2}}\right)=\frac{1}{2}\left[\left(\Sigma_{\mathrm{XX}}+\kappa \mathbf{I}\right)^{-\frac{1}{2}}-\left(\Sigma_{\mathrm{XX}}\right.\right. \\
\left.+\kappa \mathbf{I})^{-\frac{1}{2}} \tilde{k}_{X}\left(\cdot, X^{\prime}\right) \otimes \tilde{k}_{X}\left(\cdot, X^{\prime}\right)\left(\Sigma_{X X}+\kappa \mathbf{I}\right)^{-\frac{1}{2}}\right],
\end{aligned}
$$

where $\kappa>0$ is a regularization coefficient, which gives an empirical estimator. Let $\left(X_{i}, Y_{i}\right)_{i=1}^{n}$ be a sample from the distribution $F_{X Y}$. The EIF of Eq.(4) at $Z^{\prime}=\left(X^{\prime}, Y^{\prime}\right)$ for all points $Z_{i}=\left(X_{i}, Y_{i}\right)$ are

$$
\begin{aligned}
& \operatorname{EIF}\left(Z^{\prime}, \rho_{j}^{2}\right)=\widehat{\operatorname{IF}}\left(Z^{\prime}, \hat{\rho}_{j}^{2}\right), \\
& \operatorname{EIF}\left(Z_{i}, Z^{\prime}, f_{j x}\right)=\widehat{\operatorname{IF}}\left(\cdot, Z^{\prime}, f_{j X}\right), \\
& \operatorname{EIF}\left(Z_{i}, Z^{\prime}, f_{j Y}\right)=\widehat{\operatorname{EIF}}\left(\cdot, Z, \widehat{f}_{j Y}\right),
\end{aligned}
$$

respectively.

For the bounded kernels the IFs or EIFs, which are stated in Eq.(4), they have the three properties: gross error sensitivity, local shift sensitivity and rejection point. But for unbounded kernels, say a linear or polynomial, the IFs are not bounded. As a consequence, the results of classical kernel CCA using the bounded kernels are less sensitive than classical kernel CCA using the unbounded kernels [6, 27].

\section{MULTIPLE KERNEL CCA}

Multiple kernel CCA seeks more than two sets of functions in the RKHSs for which the correlation (Corr) of random variables is maximized. Given $p$ sets of random variables $X_{1}, \cdots X_{p}$ and $p$ functions in the RKHS, $f_{1}(\cdot) \in \mathcal{H}_{1}, \cdots$, $f_{p}(\cdot) \in \mathcal{H}_{p}$, the optimization problem of the random variables $f_{1}\left(X_{1}\right), \cdots, f_{p}\left(X_{p}\right)$ is

$$
\max _{\substack{f_{1} \in \mathcal{H}_{X_{i}, \cdots, f_{p} \in \mathcal{H}_{X_{i}}} \\ f_{1} \neq 0, \cdots, f_{p} \neq 0}} \sum_{j=1, j^{\prime}>j}^{p} \operatorname{Corr}\left(f_{j}\left(X_{j}\right), f_{j^{\prime}}\left(X_{j}^{\prime}\right)\right) .
$$

Given an i.i.d sample, $\left(X_{i 1}, X_{i 2}, \cdots, X_{i p}\right)_{i=1}^{n}$ from a joint distribution $F_{X_{1}, \cdots, X_{p}}$, by taking the inner products with elements or "parameters" in the RKHS, we have features 


$$
\begin{array}{r}
f_{1}(\cdot)=\left\langle f_{1}, \Phi_{1}\left(X_{1}\right)\right\rangle_{\mathcal{H}_{1}}=\sum_{i=1}^{n} a_{i 1} k_{1}\left(\cdot, X_{i 1}\right), \\
\vdots \\
f_{p}(\cdot)=\left\langle f_{p}, \phi_{p}\left(X_{p}\right)\right\rangle_{\mathcal{H}_{p}}=\sum_{i=1}^{n} a_{i p} k_{p}\left(\cdot, X_{i p}\right),
\end{array}
$$

where $k_{1}\left(\cdot, X_{1}\right), \cdots, k_{p}\left(\cdot, X_{p}\right)$ are the associated kernel functions for $\mathcal{H}_{1}, \cdots, \mathcal{H}_{p}$, respectively. The kernel Gram matrices are defined as $\mathbf{K}_{1}:=\left(k_{1}\left(X_{i 1}, X_{i^{\prime} 1}\right)\right)_{i, i^{\prime}=1}^{n}, \cdots, \mathbf{K}_{p} Y:=$ $\left(k_{1}\left(X_{i p}, X_{i^{\prime} p}\right)\right)_{i, i^{\prime}=1}^{n}$. Similar to Section 2.4, using this kernel Gram matrices, the centered kernel Gram matrices are defined as $\mathbf{M}_{1}=\mathbf{C K}_{1} \mathbf{C}, \cdots, \mathbf{M}_{p}=\mathbf{C K}_{p} \mathbf{C}$, where $\mathbf{C}=$ $\mathbf{I}_{n}-\frac{1}{n} \mathbf{B}_{n}$ with $\mathbf{B}_{n}=\mathbf{1}_{n} \mathbf{1}_{n}^{T}$ and $\mathbf{1}_{n}$ is the vector with $n$ ones. As in the two sets of data the empirical estimate of Eq. (6) is obtained using the generalized eigenvalue problem, as given by following problem:

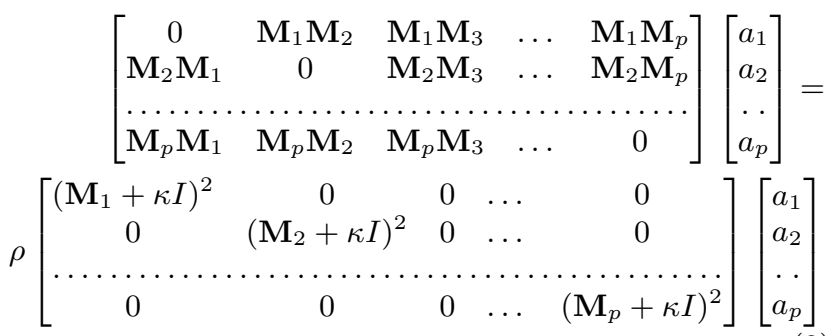

Using the similar procedure as shown in [6] and in Eq. (4) to Eq. (8), we can easily derive the IF of multiple kernel CCA. The IF of $l$-th multiple kernel CCA at $Z^{\prime}=$ $\left(X_{1}^{\prime}, \cdots, X_{P}^{\prime}\right)$ is expressed as

$$
\operatorname{IF}\left(\mathrm{Z}^{\prime}, \rho_{\mathrm{l}}^{2}\right)=-\rho_{\mathrm{l}}^{2} \sum_{\mathrm{j}=1}^{\mathrm{p}} \overline{\mathrm{f}}_{\mathrm{lj}}^{2}\left(\mathrm{X}_{\mathrm{j}}^{\prime}\right)+2 \sum_{\mathrm{j}=1, \mathrm{j}^{\prime}>\mathrm{j}}^{\mathrm{p}} \rho_{\mathrm{j}} \overline{\mathrm{f}}_{\mathrm{lj}}\left(\mathrm{X}_{\mathrm{j}}^{\prime}\right) \overline{\mathrm{f}}_{\mathrm{lj}^{\prime}}\left(\mathrm{X}_{\mathrm{j}^{\prime}}^{\prime}\right)
$$

\section{EXPERIMENTS}

We demonstrate the experiments on synthesized and real imaging genetics data analysis including SNP, fMRI, and DNA methylation. For synthesized experiments, we generate two types of data: original data and those with $5 \%$ of contamination, which are called ideal data (ID) and contaminated data (CD), respectively. In all experiments, for the bandwidth of Gaussian kernel we use the median of the pairwise distance $[22,41]$. Since the goal is to find the outlier, the regularization parameter of kernel CCA is set as $\kappa=10^{-5}$. The description of real data sets is in Sections 4.2 and the synthetic data sets are described as follows:

Multivariate Gaussian structural data (MGSD): Given multivariate normal data, $\mathbf{Z}_{i} \in \mathbb{R}^{12} \sim \mathbf{N}(\mathbf{0}, \Sigma)(i=$ $1,2, \ldots, n)$ where $\Sigma$ is the same as in [7]. We divide $\mathbf{Z}_{i}$ into two sets of variables $\left(\mathbf{Z}_{i 1}, \mathbf{Z}_{i 2}\right)$, and use the first 6 variables of $\mathbf{Z}_{i}$ as $X$ and perform $\log$ transformation of the absolute value of the remaining variables $\left.\left(\log _{e}\left(\left|\mathbf{Z}_{i 2}\right|\right)\right)\right)$ as $Y$. For the $\mathrm{CD} \mathbf{Z}_{i} \in \mathbb{R}^{12} \sim \mathbf{N}(\mathbf{1}, \Sigma)(i=1,2, \ldots, n)$.

Sign and cosine function structural data (SCSD): We use uniform marginal distribution, and transform the data by two periodic sin and cos functions to make two sets $X$ and $Y$, respectively, with additive Gaussian noise: $Z_{i} \sim$ $U[-\pi, \pi], \eta_{i} \sim N\left(0,10^{-2}\right), \quad i=1,2, \ldots, n, X_{i j}=\sin \left(j Z_{i}\right)+$ $\eta_{i}, Y_{i j}=\cos \left(j Z_{i}\right)+\eta_{i}, j=1,2, \ldots, 100$. For the CD $\eta_{i} \sim$ $N\left(1,10^{-2}\right)$.

SNP and fMRI structural data (SMSD): Two sets of SNP data X with 1000 SNPs and fMRI data Y with 1000 voxels were simulated. To correlate the SNPs with the voxels, a latent model is used as in [35]). For data contamination, we consider the signal level, 0.5 and noise level, 1 to 10 and 20 , respectively.

In the experiments, first, for the effect of kernel CCA we compared ID with CD. To measure the influence, we calculated the ratio between ID and CD of IF of kernel CC and kernel CV. Based on this ratio, we define two measures for kernel CC and kernel CV

$$
\begin{aligned}
& \eta_{\rho}=\left|1-\frac{\left\|E I F\left(\cdot, \rho^{2}\right)^{I D}\right\|_{F}}{\left\|\operatorname{EIF}\left(\cdot, \rho^{2}\right)^{C D}\right\|_{F}}\right| \quad \text { and } \\
& \eta_{f}=\left|1-\frac{\left\|E I F\left(\cdot, f_{X}\right)^{I D}-E I F\left(\cdot, f_{Y}\right)^{I D}\right\|_{F}}{\left\|E I F\left(\cdot, f_{X}\right)^{C D}-E I F\left(\cdot, f_{Y}\right)^{C D}\right\|_{F}}\right|,
\end{aligned}
$$

respectively. The method does not depend on the contaminated data, and the above measures, $\eta_{\rho}$ and $\eta_{f}$, should be approximately zero. In other words, the best methods should give smallest values. To compare, we consider simulated data sets: MGSD, SCSD, SMSD with 3 sample sizes, $n \in\{100,500,1000\}$. For each sample size, we repeat the experiment for 100 samples. Table 1 presents the results (e.g., mean \pm standard deviation) of kernel CCA. From this table, we observe that kernel CCA is affected by the contaminated data in all cases.

\subsection{Visualizing influential observation using ker- nel CCA and multiple kernel CCA}

Now, we propose a simple graphical display based on the EIF of kernel CCA, and the index plots (the data on $x$-axis and the influence of observation, as shown in Eq. (5) on $y$ axis), to assess the related influence data points in data integration with respect to EIF of kernel CCA. To do this, we first consider simulated SMSD and then real imaging genomic dataset (see 4.2). The index plots of 500 observations using the SMSD (ID and 5\% CD) and influence functions based on the EIF of kernel CCA are presented in Figure 1. The plots show that the influence of ID and CD has significance difference. On the one hand, the observations only for ID have less influence; on the other hand, the observations with $\mathrm{CD}$ have large influence. It is clear that the kernel CCA is affected by the CD significantly. In addition, using the visualization of the EIF of kernel CCA, we can easily identify the influence observations properly.

\subsection{Real data Analysis: Mind Clinical Imag- ing Consortium}

The Mind Clinical Imaging Consortium (MCIC) has collected three types of data (SNPs, fMRI and DNA methylation) from 208 subjects including 92 schizophrenic patients (age: $34 \pm 11,22$ females) and 116 (age: $32 \pm 11,44$ females) healthy controls. Without missing data, the number of subjects is 184 (81 schizophrenia (SZ) patients and 103 healthy controls)[31].

SNPs: For each subject (SZ patients and healthy controls) a blood sample was taken and DNA was extracted. All subject genes typing was performed at the Mind Research Network using the Illumina Infinium HumanOmni1- Quad assay covering 1140419 SNP loci. To form the final genotype calls and to perform a series of standard quality control 
Table 1: The mean and standard deviation of the measures, $\eta_{\rho}$ and $\eta_{f}$ of kernel CC and kernel CV.

\begin{tabular}{llcc}
\hline \multirow{2}{*}{ Data } & $\mathrm{n}$ & \multicolumn{2}{c}{ Measure } \\
\cline { 3 - 4 } MGSD & 100 & $1.9114 \pm 3.5945$ & $1.3379 \pm 3.5092$ \\
& 1000 & $1.1365 \pm 1.9545$ & $0.8631 \pm 1.3324$ \\
& $1.1695 \pm 1.6264$ & $0.6193 \pm 0.7838$ \\
\hline \multirow{2}{*}{ SCSD } & 500 & $0.2581 \pm 0.2101$ & $1.3933 \pm 1.9546$ \\
& 1000 & $0.1537 \pm 0.1272$ & $1.6822 \pm 2.2284$ \\
\hline \multirow{3}{*}{ SMSD } & 100 & $0.6455 \pm 0.0532$ & $0.6507 \pm 0.2589$ \\
& 500 & $0.6449 \pm 0.0223$ & $3.7345 \pm 2.2394$ \\
& 1000 & $0.6425 \pm 0.0134$ & $7.7497 \pm 1.2857$ \\
\hline
\end{tabular}

Table 2: The mean and standard deviation of the differences between training and testing correlation of 10 fold cross-validation kernel CCA.

\begin{tabular}{cccc}
\hline Data & $\mathrm{n}$ & $\mathrm{ID}$ & $\mathrm{CD}$ \\
\hline \multirow{3}{*}{ MGSD } & 500 & $0.7005 \pm 0.0744$ & $0.7536 \pm 0.0503$ \\
& 1000 & $0.6459 \pm 0.0234$ & $0.5322 \pm 0.1184$ \\
& 2000 & $0.4151 \pm 0.210$ & $0.4673 \pm 0.1196$ \\
SCSD & 500 & $0.3601 \pm 0.3132$ & $0.2974 \pm 0.3433$ \\
& 1000 & $0.0005 \pm 0.0 .0002$ & $0.0 .0003 \pm 0.0 .0005$ \\
& 2000 & $0.0002 \pm 0.0 .0001$ & $0.0 .0003 \pm 0.0 .0002$ \\
\hline
\end{tabular}

Table 3: The stem-and-leaf display of the influence MCIC data (the decimal point is 4 digits for pairwise data and 2 digits for 3 data sets) using kernel CCA and multiple kernel CCA.

\begin{tabular}{|c|c|c|c|c|c|c|c|}
\hline \multicolumn{2}{|c|}{ SNP \& fMRI } & \multicolumn{2}{|c|}{ SNP \& Methylation } & \multicolumn{2}{|c|}{ fMRI \&Methylation } & \multicolumn{2}{|r|}{3 Data sets } \\
\hline 0 & $00000000+35$ & 0 & $00000000+77$ & $\overline{0 \mid}$ & $00000000+46$ & 0 & $00000000+147$ \\
\hline $1 \mid$ & $00111223+1$ & $1 \mid$ & $00012223+7$ & $1 \mid$ & 01122256 & $1 \mid$ & 01122345 \\
\hline $2 \mid$ & 01223334 & $2 \mid$ & $01122223+2$ & 2 & 01222333 & 2 & 36 \\
\hline 3 & 11234557 & 3 & 11112456 & 3 & 01113378 & 3 & 9 \\
\hline 4 & 03357788 & $4 \mid$ & 1223557 & $4 \mid$ & 2333455 & 4 & \\
\hline 5 & 01367788 & 5 & 2 & $5 \mid$ & 11123567 & $\vdots$ & \\
\hline $6 \mid$ & 00012334 & 6 & 158 & 6 & 1345668 & $\vdots$ & \\
\hline $7 \mid$ & 02455779 & $7 \mid$ & 2 & $7 \mid$ & 0568 & $\vdots$ & \\
\hline $8 \mid$ & 446799 & $8 \mid$ & 2 & $8 \mid$ & 3334778 & $\vdots$ & \\
\hline $9 \mid$ & 14 & : & & $9 \mid$ & 248 & $\vdots$ & \\
\hline 10 & 0016799 & 32 & 8 & 10 & 036699 & $\vdots$ & \\
\hline : & & 34 & 9 & : & & $\vdots$ & \\
\hline $21 \mid$ & 7 & 36 & 0 & 36 & 6 & $13 \mid$ & 6 \\
\hline 22 & 59 & $37 \mid$ & 4 & 41 & 2 & & \\
\hline 25 & 39 & 40 & 8 & 44 & 2 & & \\
\hline 29 & 5 & 62 & 6 & 49 & 2 & & \\
\hline 30 & 9 & 72 & 0 & 60 & 7 & & \\
\hline $31 \mid$ & 3 & 90 & 7 & 77 & 0 & & \\
\hline
\end{tabular}




\section{Ideal data}

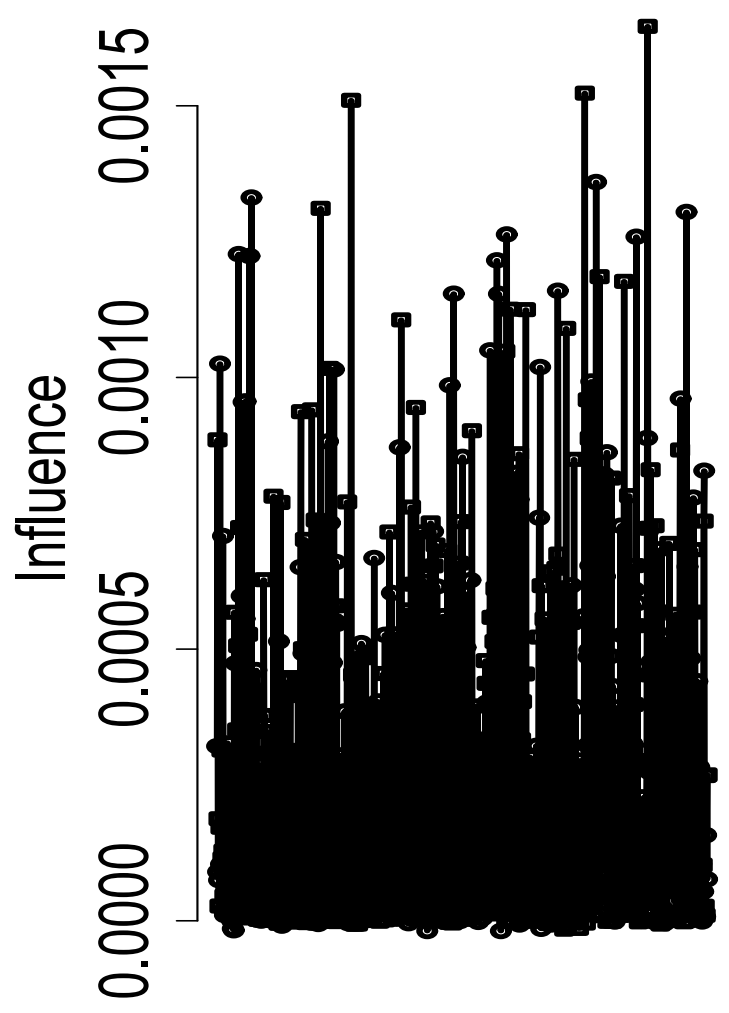

Index
Contaminated data

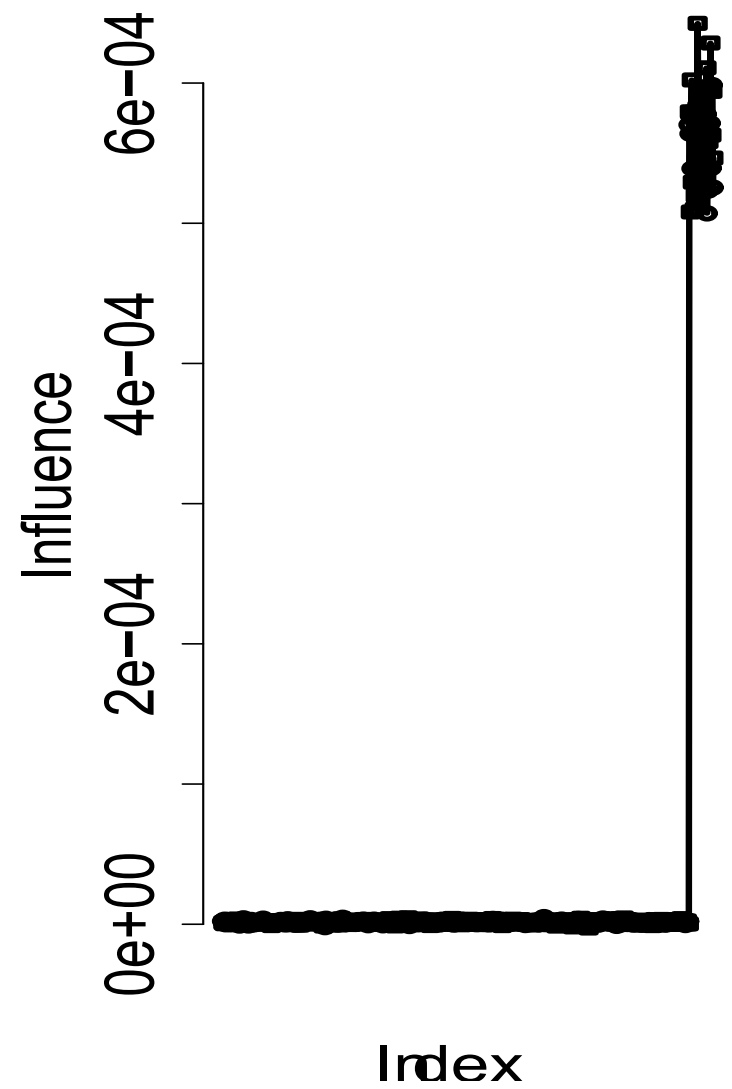

Figure 1: Influence points using empirical influence function of kernel CCA of of SMSD (ideal data and contaminated data).

Table 4: Mean and standard deviation of the differences between taring and test correlation of 10 fold cross-validation of MICI data using kernel CCA and multiple kernel CCA.

\begin{tabular}{llcc}
\hline Data & outliers & All & Without outliers \\
\hline SNP \& fMRI & $\{135,94,7,4,169,67,97,9\}$ & $0.8208 \pm 0.2382$ & $0.7353 \pm 0.1870$ \\
SNP \& Methylation & $\{67,12,85,3,7,9,14,92\}$ & $0.7337 \pm 0.2000$ & $0.6606 \pm 0.1772$ \\
fMRI \&Methylation & $\{30,99,13,67,141,101\}$ & $0.7424 \pm 0.1893$ & $0.7817 \pm 0.1759$ \\
SNP, fMRI \& Methylation & $\{67,30,99,7\}$ & $1.4115 \pm 0.2544$ & $0.1 .2659 \pm 0.1744$ \\
\hline
\end{tabular}



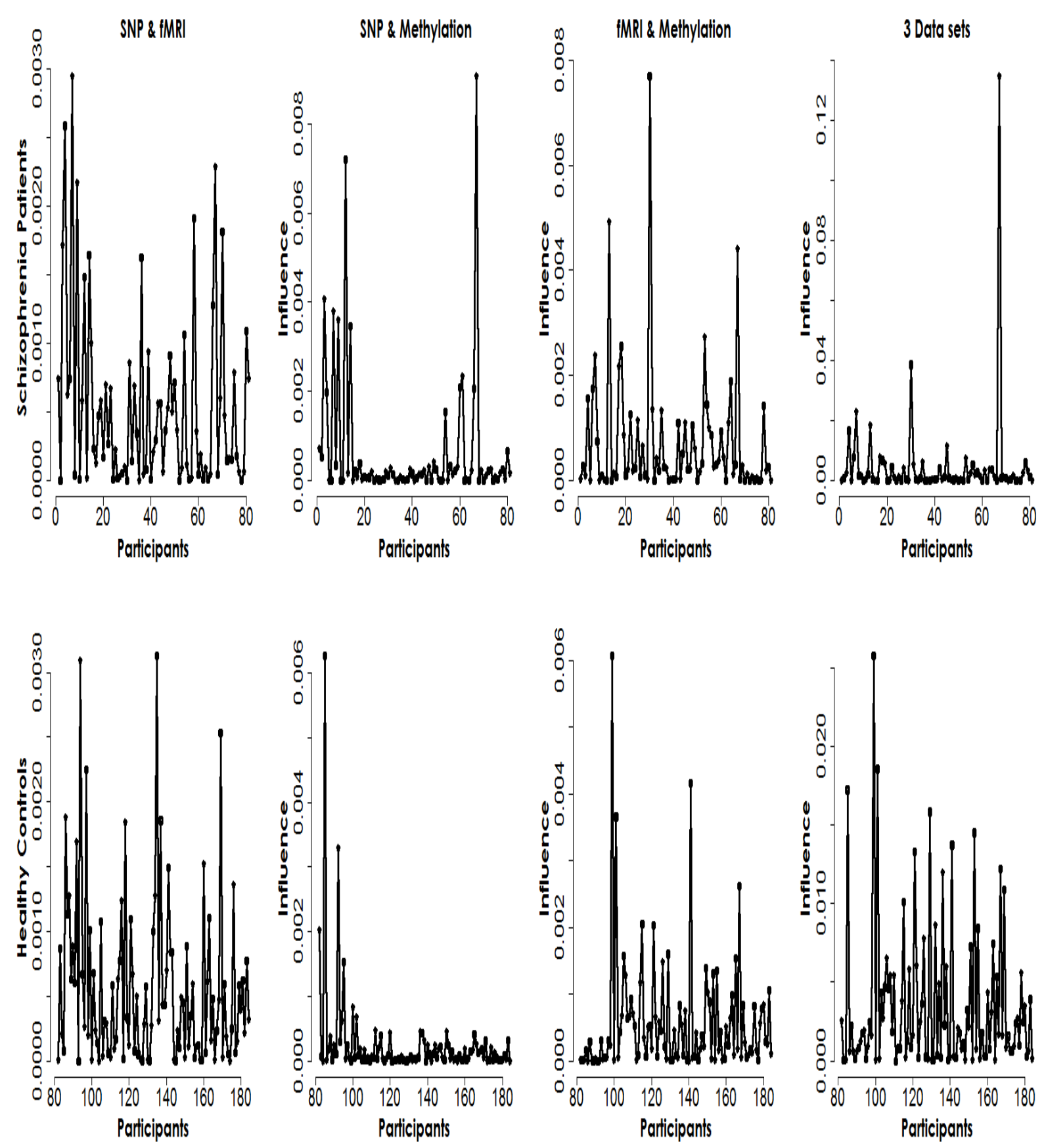

Figure 2: The influence subject of MCIC data set using kernel CCA and multiple kernel CCA. 
procedures bead studio and PLINK software packages were applied, respectively. The final dataset spans 722177 loci having 22442 genes based on 184 subjects (those without missing data). Genotypes "aa" (non-minor allele), "Aa" (one minor allele) and "AA" (two minor alleles) were coded as 0 , 1 and 2for each SNP, respectively [31] [12].

fMRI: Participants' fMRI data was collected during their block design motor response to auditory stimulation. Stateof-the-art approaches use mainly Participants' feedback and experts' observations for this purpose. The aim was to continuously monitor the patients, acquiring images with parameters $(\mathrm{TR}=2000 \mathrm{~ms}, \mathrm{TE}=30 \mathrm{~ms}$, field of view $=22 \mathrm{cam}$, slice thickness $=4 \mathrm{~mm}, 1 \mathrm{~mm}$ skip, 27 slices, acquisition matrix $64 \times 64$, flip angle $=90^{\circ}$ ) on a Siemens3T Trio Scanner and 1.5 T Sonata with echo-planar imaging (EPI). Data were pre-processed with SPM5 software and were realigned spatially normalized and resliced to $3 \times 3 \times 3 \mathrm{~mm}$. It was smoothed with a $10 \times 10 \times 10 \mathrm{~mm}^{3}$ Gaussian kernel and analyzed by multiple regression considering the stimulus and their temporal derivatives plus an intercept term as repressors . Finally the stimulus-on versus stimulus-off contrast images were extracted with $53 \times 63 \times 46$ mission measurements, excluding voxels without measurements. 41236 voxels were extracted from 116 ROIs based on the aal brain atlas for analysis [31].

DNA methylation:DNA methylation is one of the main epigenetic mechanisms to regulate gene expression. It appears to be involved in the development of SZ. In this paper, we investigated 27481 DNA methylation markers in blood from 81 SZ patients and 103 healthy controls. Participants come from the MCIC, a collaborative effort of 4 research sites. For more details, site information and enrollment for SZ patients and healthy controls are in [32]. All participants' symptoms were evaluated by the Scale of the Assessment of Positive Symptoms and the Scale of the Assessment of Negative symptoms [9]. DNA from blood samples was measured by the Illumina Infinium Methylation27 Assay. The methylation value is calculated by taking the ratio of the methylated probe intensity and the total probe intensity.

To detect influential subjects (in SZ patients and healthy controls), as discussed in Section 4.1, we use the EIF of kernel CC of kernel CCA and multiple kernel CCA. Figure 2 shows the influence of participants from MICI data: SNPs, fMRI and DNA methylation. The SZ patients and healthy controls are in 1 st and 2 nd rows, respectively. The analysis results of pairwise data sets (i.e., SNP \& fMRI, SNP \& Methylation, and fMRI\& Methylation) using kernel CCA and all 3 data sets, SNP, fMRI, \& Methylation using multiple kernel CCA are in column 1st to 4 th, respectively. These plots show that in all scenarios the healthy controls have less influence than the SZ patients group.

To extract the outliers of subjects from participants of MCIC data, we consider stem-and-leaf display of influence of MCIC data (e.g., SNP \& fMRI, SNP \& Methylation, and fMRI\& Methylation) using kernel CCA and all 3 data sets, SNP, fMRI, \& Methylation using multiple kernel CCA. Table 2 shows the results of pairwise datasets and 3 datasets together. Based on the stem-and-leaf display, the outliers of subject sets of SNP \& fMRI, SNP \& Methylation, and fMRI\& Methylation, and SNP, fMRI, \& Methylation are $\{135,94,7,4,169,67,97,9\},\{67,12,85,3,7,9,14,92\}$, $\{30,99,13,67,141,101\},\{67,30,99,7\}$. It is noted that, multiple kernel CCA is able to extract common SZ patient 67 , which is also outlier for all pairwise results using kernel CCA. Finally, we investigated the difference between training correlation and test correlation using 10 fold cross-validation with all subjects with or without outliers. Table 4 shows the outliers of subjects along with the result of all subjects with or without outliers using kernel CCA and multiple kernel CCA. We see that after removing the outliers by the proposed methods, both kernel CCA and multiple kernel CCA performed better using all subjects.

\section{CONCLUDING REMARKS AND FUTURE RESEARCH}

The methods for identifying outliers in imaging genetics data presented in this paper are not only applicable to single data sets but also for integrated data sets, which is an essential and challenging issue for multiple sources data analysis. The proposed methods are based on the IF of kernel CCA and multiple kernel CCA, which can detect and isolate the outlier effectively in both synthesized and real data sets. After applying to pairwise data (e.g., SNP \& fMRI, SNP \& Methylation, and fMRI\& Methylation) using kernel CCA and to all 3 data sets (e.g., SNP, fMRI, \& Methylation) using multiple kernel CCA, we found that in all scenarios the healthy controls have less influence than the SZ patients. In addition, multiple kernel CCA is able to extract the common SZ patient 67 , which is also the outliers for all pairwise data analysis using kernel CCA. After removing the significant outliers indicated by both kernel CCA and multiple kernel CCA, the stem-and-leaf display shows that both methods performed much better than using all subjects.

Although we have argued that the kernel CCA and multiple kernel CCA procedure for detecting outliers worked effectively, there is also space for further improvement. The use of the Gaussian kernel function is an optimal selection; however, other classes of kernel functions may be more reasonable for a specific data set. In future work, it would be also interesting to develop robust kernel PCA and robust multiple kernel CCA and apply them to imaging genomic analysis.

\section{Acknowledgments}

We would like to thank the reviewers for their careful reading of the manuscript and their useful comments. The authors also wish to thank the NIH (R01 GM109068, R01 MH104680) and NSF (1539067) for support.

\section{REFERENCES}

[1] S. Akaho. A kernel method for canonical correlation analysis. International meeting of psychometric Society., 35:321-377, 2001.

[2] M. A. Alam. Kernel Choice for Unsupervised Kernel Methods. PhD. Dissertation, The Graduate University for Advanced Studies, Japan, 2014.

[3] M. A. Alam and K. Fukumizu. Higher-order regularized kernel CCA. 12th International Conference on Machine Learning and Applications, pages 374-377, 2013.

[4] M. A. Alam and K. Fukumizu. Hyperparameter selection in kernel principal component analysis. Journal of Computer Science, 10(7):1139-1150, 2014.

[5] M. A. Alam and K. Fukumizu. Higher-order regularized kernel canonical correlation analysis. 
International Journal of Pattern Recognition and Artificial Intelligence, 29(4):1551005(1-24), 2015.

[6] M. A. Alam, K. Fukumizu, and Y.-P. Wang. Robust Kernel (Cross-) Covariance Operators in Reproducing Kernel Hilbert Space toward Kernel Methods. ArXiv e-prints, Feb. 2016.

[7] M. A. Alam, M. Nasser, and K. Fukumizu. Sensitivity analysis in robust and kernel canonical correlation analysis. 11th International Conference on Computer and Information Technology, Bangladesh., IEEE:399-404, 2008.

[8] M. A. Alam, M. Nasser, and K. Fukumizu. A comparative study of kernel and robust canonical correlation analysis. Journal of Multimedia., 5:3-11, 2010.

[9] N. Andreasen. Scale for the assessment of positive symptoms (SAPS). Springer, Iowa City, University of Iowa, 1984.

[10] N. Aronszajn. Theory of reproducing kernels. Transactions of the American Mathematical Society, 68:337-404, 1950.

[11] F. R. Bach and M. I. Jordan. Kernel independent component analysis. Journal of Machine Learning Research, 3:1-48, 2002.

[12] X. Chen and H. Liu. An efficient optimization algorithm for structured sparse cca, with applications to eqtl mapping. Statistics in BioSciences, 2012.

[13] A. Christmann and I. Steinwart. On robustness properties of convex risk minimization methods for pattern recognition. Journal of Machine Learning Research, 5:1007-1034, 2004.

[14] A. Christmann and I. Steinwart. Consistency and robustness of kernel-based regression in convex risk minimization. Bernoulli, 13(3):799-819, 2007.

[15] D. L. Donoho and P. J. Huber. The notion of breakdown point. In P. J. Bickel, K. A. Doksum, and J. L. Hodges Jr, editors, A Festschrift for Erich L. Lehmann, Belmont, California, Wadsworth, 12(3):157-184, 1983.

[16] P. Filzmoser, R. Maronna, and M. Werner. Outlier identification in high dimensions. computational Stastisticses Data Analysis, 52(2008):1694-1711, 2008.

[17] K. Fukumizu, F. R. Bach, and A. Gretton. Statistical consistency of kernel canonical correlation analysis. Journal of Machine Learning Research, 8:361-383, 2007.

[18] K. Fukumizu, A. Gretton, X. Sun, and B. Schölkopf. Kernel measures of conditional dependence. In Advances in Neural Information Processing Systems, Cambridge, MA, MIT Press, 20:489-496, 2008.

[19] K. Fukumizu and C. Leng. Gradient-based kernel dimension reduction for regression. Journal of the American Statistical Association, 109(550):359-370, 2014.

[20] P. Gogoi, D. Bhattacharyya, B. Borah, and J. K. Kalita. A survey of outlier detection methods in network anomaly identification. The Computer Journal, 54(4):570-588, 2011.

[21] A. Gretton, K. M. Borgwardt, M. J. Rasch, B. Schölkopf, and A. J. Smola. A kernel two-sample test. Journal of Machine Learning Research, 13:723773, 2012.

[22] A. Gretton, K. Fukumizu, C. H. Teo, L. Song, B. Schölkopf, and A. Smola. A kernel statistical test of independence. In Advances in Neural Information Processing Systems, 20:585-592, 2008.

[23] F. R. Hampel. The influence curve and its role in robust estimations. Journal of the American Statistical Association, 69:386-393, 1974.

[24] F. R. Hampel, P. J. R. E. M. Ronchetti, and W. A. Stahel. Robust Statistics: The Approach Based on Influence Functions. John Wiley \& Sons, New York, 2011.

[25] F. R. Hampel, E. M. Ronchetti, and W. A. Stahel. Robust Statistics. John Wiley \& Sons, New York, 1986.

[26] T. Hofmann, B. Schölkopf, and J. A. Smola. Kernel methods in machine learning. The Annals of Statistics, 36:1171-1220, 2008.

[27] S. Y. Huang, Y. R. Yeh, and S. Eguchi. Robust kernel principal component analysis. Neural Computation, 21(11):3179-3213, 2009.

[28] P. J. Huber. Robust estimation of a location parameter. Annals of Mathematical Statistics, 35:73-101, 1964.

[29] P. J. Huber and E. M. Ronchetti. Robust Statistics. John Wiley \& Sons, England, 2009.

[30] J. Kim and C. D. Scott. Robust kernel density estimation. Journal of Machine Learning Research, 13:2529-2565, 2012.

[31] D. Lin, V. D. Callhoun, and Y. P. Wang. Correspondence between fmri and snp data by group sparse canonical correlation analysis. Medical Image Analysis, 18:891 - 902, 2014.

[32] J. Liu, J. Chen, S. Ehrlich, E.Walton, T. W. N. P.Bizzozero, J. Bustillo, J. A. Turner, and V. D. Calhoun. Methylation patterns in whole blood correlate with symptoms in schizophrenia patients. Schizophrenia Bulletin, 40(4):769-776, 2014.

[33] M. Naser and M. A. A. N. A. Hamzah. Qualitative robustness in estimation. Pakistan Journal of Statistics and Operation Research, 8(3):619-634, 2012.

[34] J. H. Oh and J. Gao. A kernel-based approach for detecting outliers of high-dimensional biological data. IEEE International conference on Bioinformatics and Biomedicine (BIBM), 10:Suppl 4:S7, 2009.

[35] E. Parkhomenko, D. Tritchler, and J. Beyene. Sparse canonical correlation analysis with application to genomic data integration. Statistical Applications in Genetics and Molecular Biolog, 8(1):1-34, 2009.

[36] M. Romanazzi. Influence in canonical correlation analysis. Psychometrika, 57(2):237-259, 1992.

[37] V. Roth. Kernel fisher discriminants for outlier detection. Neural Computation, 18(4):942-960, 2006.

[38] B. D. M. S. Yu, L-C. Tranchevent and Y. Moreau. Kernel-based Data Fusion for Machine Learning. Springer, Verlag Berlin Heidelberg, 2011.

[39] B. Schölkopf, A. J. Smola, and K.-R. Müller. Nonlinear component analysis as a kernel eigenvalue problem. Neural Computation., 10:1299-1319, 1998.

[40] L. Song, A. Smola, K. Borgwardt, and A. Gretton. Colored maximum variance unfolding. Advances in 
Neural Information Processing Systems, 20:1385-1392, 2008.

[41] T. Sun and S. Chen. Locality preserving cca with applications to data visualization and pose estimation. Image and Vision Computing, 25 (5):531-543, 2007.

[42] J. W. Tukey. Exploratory Data Analysis. Addison-Wesley, Reading, Massachusetts, 1977. 